\title{
HIF-Prolyl Hydroxylase Inhibitors: Confirmed Efficacy with Uncertain Safety
}

\author{
Simon D. Roger ${ }^{a}$ Daniel W. Coyne ${ }^{b}$ \\ ${ }^{a}$ Department of Renal Medicine, Gosford Hospital, Gosford, NSW, Australia; ${ }^{b}$ Department of Medicine, Washington \\ University in St. Louis, St. Louis, MO, USA
}

Treatment options for the anemia of chronic kidney disease (CKD) continue to expand. Several hypoxia-inducible factor-prolyl hydroxylase inhibitors (HIF-PHIs) are undergoing clinical testing and seeking regulatory approval. Erythropoiesis-stimulating agents (ESAs) have been the cornerstone of anemia therapy since 1989, when first-in-class recombinant human erythropoietin was approved. The rise in hemoglobin $(\mathrm{Hb})$ with ESAs demanded greater need for iron and paradoxically greater iron losses due to the higher $\mathrm{Hb}$ concentration. This exacerbated iron deficiency led to markedly increased use of intravenous (IV) iron. ESAs, especially at larger dosages aimed to target higher target $\mathrm{Hb}$ levels, increase the risk of cardiovascular and thrombotic events, cancer, and death. Prescribed IV iron therapy, while apparently safer, has limited long-term safety evidence and fills iron stores well beyond the normal range, which in part overcomes hepcidin-mediated iron blockade.

In this issue of the journal, Yamamoto and colleagues $[1,2]$ published 2 trials investigating the efficacy and safety of the HIF-PHI, molidustat, in Japanese stage 3-5 nondialysis patients with anemia of CKD. Each dose of HIFPHI stimulates a pulse of endogenous erythropoietin, upregulates iron transporters, and enhances iron release from stores. These actions indirectly suppress hepcidin, the master regulator of iron absorption [3]. HIF-PHIs stimulate and maintain erythropoiesis and, in some trials, reduce the need for IV iron supplementation. HIF-PHIs could supplant ESAs as the cornerstone treatment for

karger@karger.com

(C) 2021 S. Karger AG, Basel

www.karger.com/ajn

Karger"
CKD-related anemia if their safety is comparable or superior to ESAs. But, proving HIF-PHIs are as safe as ESAs is a giant hurdle.

Yamamoto and colleagues [1] report results from two 52-week treatment trials: an anemia correction trial in ESA-naïve subjects and an $\mathrm{Hb}$ maintenance trial in subjects already receiving an ESA [2]. All subjects had CKD nondialysis stage $3-5$ with anemia and were randomized 1:1 to daily oral molidustat or subcutaneous darbepoetin alfa. In both trials, drug doses were titrated by protocols to achieve and maintain an $\mathrm{Hb}$ of $\geq 11.0 \mathrm{~g} / \mathrm{dL}$ to $<13.0 \mathrm{~g} /$ $\mathrm{dL}$. These higher target $\mathrm{Hb}$ concentrations were chosen based on the Japanese Society for Dialysis Therapy guidelines [4], whereas in Western nephrology, the Kidney Disease Improving Global Outcomes recommend maintaining $\mathrm{Hb} \leq 11.5 \mathrm{~g} / \mathrm{dL}$ [5].

In the anemia correction trial, 162 patients were randomized to $25 \mathrm{mg}$ of molidustat orally daily or $30 \mu \mathrm{g}$ weekly of darbepoetin alfa subcutaneously. In the $\mathrm{Hb}$ maintenance trial, 164 subjects receiving an ESA with an $\mathrm{Hb}$ level of $\geq 10.0 \mathrm{~g} / \mathrm{dL}$ and $<13.0 \mathrm{~g} / \mathrm{dL}$ were randomized to either $25 \mathrm{mg}$ or $50 \mathrm{mg}$ of molidustat daily (depending on their prior ESA requirement) or were initiated on darbepoetin alfa at a starting dose in accordance with the previous ESA dose and injected subcutaneously once every 2 or 4 weeks.

For both trials, molidustat was noninferior to darbepoetin alfa for correcting and maintaining $\mathrm{Hb}$ in the target range. In the maintenance trial, mean $\mathrm{Hb}$ during the eval- 
Table 1. Adjudicated MACE in molidustat trials

\begin{tabular}{|c|c|c|c|c|c|c|}
\hline \multirow[t]{2}{*}{ Event } & \multicolumn{2}{|c|}{ Anemia correction trial } & \multicolumn{2}{|c|}{ Anemia maintenance trial } & \multicolumn{2}{|c|}{$\begin{array}{l}\text { Cumulative safety data in molidustat } \\
\text { anemia correction and maintenance trial }\end{array}$} \\
\hline & $\begin{array}{l}\text { molidustat } \\
(N=82)\end{array}$ & $\begin{array}{l}\text { darbepoetin } \\
(N=79)\end{array}$ & $\begin{array}{l}\text { molidustat } \\
(N=82)\end{array}$ & $\begin{array}{l}\text { darbepoetin } \\
(N=82)\end{array}$ & $\begin{array}{l}\text { molidustat } \\
(N=164)\end{array}$ & $\begin{array}{l}\text { darbepoetin } \\
(N=161)\end{array}$ \\
\hline Patients with $\geq 1$ MACE, $n$ & 6 & 0 & 3 & 1 & 9 & 1 \\
\hline Death & $2^{*}$ & 0 & 2 & 0 & $4^{*}$ & 0 \\
\hline Myocardial infarction & 2 & 0 & 1 & 0 & 3 & 0 \\
\hline Stroke & 3 & 0 & 0 & 1 & 3 & 1 \\
\hline
\end{tabular}

MACE included undetermined death, myocardial infarction, unstable angina, ischemic stroke, pulmonary thromboembolism, and acute limb ischemia. Data are from the safety analysis set as shown in tables 9[1] and 3[2] in the supplements. MACE, major adverse cardiovascular events. ${ }^{*}$ There were 3 deaths in the molidustat group of the anemia correction trial. Exclusion of 1 death from this table is likely due to these trials using cardiovascular deaths, not all-cause deaths, as a component of the MACE criteria.

uation period was $11.67 \mathrm{~g} / \mathrm{dL}$ (95\% confidence interval [CI]: $11.48-11.85)$ in the molidustat group versus $11.53 \mathrm{~g} /$ dL (95\% CI: 11.31-11.74) in the darbepoetin group.

In the anemia correction trial of ESA-naïve patients, mean $\mathrm{Hb}$ in the molidustat group during the week 30-36 evaluation period was $11.28 \mathrm{~g} / \mathrm{dL}$ (95\% CI: 11.07-11.50) versus $11.70 \mathrm{~g} / \mathrm{dL}$ (95\% CI: 11.50-11.90) for the darbepoetin alfa group. Molidustat was noninferior to darbepoetin in the change in mean $\mathrm{Hb}$ level during the evaluation period from baseline, $-0.38(-0.67,-0.08) \mathrm{g} / \mathrm{dL}$. Previous ESA trials and current HIF-PI trials have used a smaller noninferiority margin $(0.75 \mathrm{~g} / \mathrm{dL})[6,7]$. During the evaluation period, $68.3 \%$ of patients in the molidustat group and $85.0 \%$ of the darbepoetin alfa group had $\mathrm{Hb}$ levels within the prespecified target range. These are somewhat lackluster efficacy data for molidustat, but certainly do not indicate inferiority, as it was a protocol-driven $\mathrm{Hb}$ target trial. As the authors state, some of these between-group differences may reflect in part more dropouts by patients randomized to molidustat. The slower $\mathrm{Hb}$ response to molidustat could be corrected in clinical practice by use of higher doses of molidustat, but it is unknown if this would increase the number of drug-related adverse events and major adverse cardiovascular events (MACE).

Overall, molidustat efficacy was noninferior to darbepoetin alfa related to $\mathrm{Hb}$ increases, and generally these results are consistent with other HIF-PHI trials in CKD nondialysis (CKD-ND) patients. As an example, 2 parallel CKD-ND trials of vadadustat in 1,751 ESA-untreated CKD-ND patients and 1,725 ESA-treated CKD-ND patients showed noninferiority for $\mathrm{Hb}$ correction and maintenance versus the active comparator, darbepoetin alfa, during week 24-36 of therapy [7]. Similarly, in the 616-pa- tient, 2-year trial of roxadustat, an $\mathrm{Hb}$ response was achieved in the first 24 weeks by $89.5 \%$ of roxadustat patients and $78.0 \%$ of darbepoetin alfa patients, and mean $\mathrm{Hb}$ was maintained $>11.0 \mathrm{~g} / \mathrm{dL}$ in both groups out to 2 years [8]. Indeed, trials of daprodustat, enarodustat, roxadustat, and vadadustat versus ESA therapy have all demonstrated noninferiority for anemia correction or maintenance versus ESA therapy [3].

The decision to choose HIF-PHIs over ESA therapy hinges on proof of safety, not efficacy. Yamamoto and colleagues [4] carefully collected all treatment emergent adverse events (TEAEs), especially MACE - which these trials define as cardiovascular death, myocardial infarction, unstable angina, ischemic stroke, pulmonary thromboembolism, and acute limb ischemia. The MACE were adjudicated by an independent committee during both of these 1-year trials, though they do not state whether these members were blinded to the patient's drug assignment.

In the anemia correction trial, confirmed MACE occurred in $7.3 \%(6 / 82)$ of patients treated with molidustat and none $(0 / 79)$ of those treated with darbepoetin alfa. There were 3 deaths in the molidustat group and no deaths with darbepoetin alfa-treated subjects. In the anemia maintenance trial, confirmed MACE occurred in $3.7 \%(3 / 82)$ of patients treated with molidustat and $1.2 \%$ $(1 / 82)$ of those treated with darbepoetin alfa. There were 2 deaths in the molidustat group and no deaths in the darbepoetin alfa group. Whether these events are attributable to the investigational product or not can be the subject of bias in an unblinded trial.

As shown in Table 1, there is an apparent imbalance in MACE in these 2 trials that raise serious safety concerns about molidustat. When the MACE are combined across 
these trials, there are 9/164 with MACE on molidustat versus $1 / 161$ patients on darbepoetin alfa. A $\chi^{2}$ test of this difference yields a nominal $p$ value of 0.01 for molidustat increasing MACE relative to darbepoetin. There are reasons to think combining the safety outcomes of these 2 trials is appropriate, including the populations, trial designs, and dosing protocols which are similar, and the data collection and safety analyses are the same. The trials even employed the same 2 specialists to adjudicate MACE. Finally, combining safety analyses across trials is common as it takes many more patients and events to assess safety. As an example, the vadadustat phase 3 trial in CKD-ND performed a safety assessment by combining TEAEs and MACE from their ESA-naïve, anemia correction trial and their ESA-treated anemia maintenance trial [7]. Although this is post hoc, the treatment period in the molidustat trials is very short (1 year), and the MACE rates are extremely low. Consequently, we cannot be confident molidustat is less safe than darbepoetin alfa. But, we should have concerns, as it took many years and large numbers of patients in the ESA clinical trials for the nephrology community to recognize the association between ESAs and MACE.

MACE appear to be increased with at least one other HIF-PHI, vadadustat, relative to ESA in large safety analyses. Two large trials of vadadustat versus darbepoetin in dialysis-dependent CKD patients show no significant increase in MACE with the HIF-PHI versus ESA (hazard ratio 0.96 [95\% CI: 0.83-1.11]) [9]. However, 2 CKD-ND trials of vadadustat did find significantly increased MACE with vadadustat compared to darbepoetin with a hazard ratio for MACE of 1.17 (95\% CI: 1.01-1.36) [7]. A subgroup analysis found the increased risk was seen in only the non-US sites, which had used an $\mathrm{Hb}$ target of 10-12 $\mathrm{g} / \mathrm{dL}$ versus $10-11 \mathrm{~g} / \mathrm{dL}$ employed at US sites. Could the higher $\mathrm{Hb}$ target of 11-13 g/dL used in these molidustat trials in Japanese patients have led the marked imbalance in MACE between trial arms? Only large outcome trials with different $\mathrm{Hb}$ targets can answer that question.

Secondly, safety data presented by Fibrogen in late 2019 at ASN Kidney Week showed roxadustat resulted in significantly less MACE (all-cause mortality and nonfatal stroke or myocardial infarction) and MACE+ (MACE and hospitalization for heart failure or unstable angina) than epoetin alfa in dialysis-dependent CKD patients, especially in incident dialysis CKD patients [10]. Almost 18 months later, Fibrogen announced the primary cardiovascular safety analyses were not the prespecified outcomes, and the MACE superiority to ESA claimed for roxadustat was simply untrue [11], when post hoc changes to stratification factors were appreciated.
There are other concerns about HIF-PHIs. As an example, in the molidustat anemia correction trial, diabetic retinopathy - a concern because HIF upregulation may worsen disease - developed in 3 molidustat-treated patients and 1 patient on darbepoetin alfa. A trial of the HIF-PHI, roxadustat, focused on diabetic retinopathy in 301 hemodialysis patients and found no increased incidence of retinopathy development or progression compared to darbepoetin [12]. Other concerns included with these agents include increased cancer risk or cancer progression and potential to exacerbate pulmonary hypertension. Trials to assess these concerns will take years to complete.

Today, there are HIF-PI drugs registered for use in Japan and China. There have been regulatory submissions in the USA and Europe or are upcoming for these agents. These are still early times in our experience with this class of drug. The phase 3 trial results provide potential signals that there are side effects of HIF-PHIs that may be class rather than drug specific. In the future, we may be treating anemia the same way diabetes is managed both with lower dosages of injectable insulin and oral medication that enhances endogenous insulin production. There is much more work to be done.

\section{Conflict of Interest Statement}

S.D.R. reports he has been an author on HIF-PHI trial articles and/or abstracts for Fibrogen and Astra Zeneca. Previously he was the country lead for GlaxoSmithKline, a HIF-PHI manufacturer. He has also been a site investigator in HIF-PHI trials for Fibrogen and GlaxoSmithKline and received speaker fees from Astra Zeneca. D.W.C. reports he is a paid consultant to the HIF-PHI manufacturers Abekia/Otsuka, GlaxoSmithKline, and Astra Zeneca and was a consultant to Fibrogen. He has been an author on HIFPHI trial articles and/or abstracts for Fibrogen and GlaxoSmithKline. He has also been a site investigator in HIF-PHI trials for Fibrogen and GlaxoSmithKline, wherein all payments accrue to his university.

\section{Funding Sources}

The authors did not receive any funding.

\section{Author Contributions}

S.D.R. conceived of the editorial, created the first draft, and revised later drafts, including the final submission. D.W.C. revised and expanded the first draft and added to later drafts. 


\section{References}

1 Yamamoto H, Nobori K, Matsuda Y, Hayashi Y, Hayasaki T, Akizawa T. Efficacy and safety of molidustat for anemia in ESA-naive nondialysis patients: a randomized, phase 3 trial. Am J Nephrol. 2021. http://dx.doi.org/ $10.1159 / 000518071$.

2 Yamamoto H, Nobori K, Matsuda Y, HayashiY, Hayasaki T, Akizawa T. Molidustat for renal anemia in non dialysis patients previously treated with erythropoiesis-stimulating agents: a randomized, open-label, phase 3 study. Am J Nephrol. 2021. http://dx.doi. org/10.1159/000518072.

3 Haase VH. Hypoxia-inducible factor-prolyl hydroxylase inhibitors in the treatment of anemia of chronic kidney disease. Kidney Int Suppl. 2021;11(1):8-25.

4 Yamamoto H, Nishi S, Tomo T, Masakane I, Saito K, Nangaku M, et al. 2015 Japanese society for dialysis therapy: guidelines for renal anemia in chronic kidney disease. Ren Replace Ther. 2017;3(1):1-46.
5 Kidney Disease: Improving Global Outcomes Anemia Work Group. KDIGO clinical practice guideline for anemia in chronic kidney disease. Kidney Int Suppl. 2012;2(4):279-335.

6 Roger SD, Locatelli F, Woitas RP, Laville M, Tobe SW, Provenzano R, et al. C.E.R.A. once every 4 weeks corrects anaemia and maintains haemoglobin in patients with chronic kidney disease not on dialysis. Nephrol Dial Transplant. 2011;26(12):3980-6.

7 Chertow GM, Pergola PE, Farag YMK, Agarwal R, Arnold S, Bako G, et al. Vadadustat in patients with anemia and non-dialysis-dependent CKD. N Engl J Med. 2021;384(17): 1589-600.

8 Barratt J, Andric B, Tataradze A, Schömig M, Reusch M, Valluri U, et al. Roxadustat for the treatment of anaemia in chronic kidney disease patients not on dialysis: a phase 3 , randomised, open-label, active-controlled study (DOLOMITES). Nephrol Dial Transplant. 2021. http://dx.doi.org/10.1093/ndt/gfab191.
9 Eckardt K-U, Agarwal R, Aswad A, Awad A, Block GA, Bacci MR, et al. Safety and efficacy of vadadustat for anemia in patients undergoing dialysis. N Engl J Med. 2021;384(17): 1601-12.

10 Provenzano R, Fishbane S, Szczech L, Leong $\mathrm{R}$, Saikali KG, Zhong M, et al. Pooled analysis of roxadustat for anemia in patients with kidney failure incident to dialysis. Kidney Int Rep. 2021;6(3):613-23.

$11 \mathrm{https} / /$ investor.fibrogen.com/news-releases/ news-release-details/fibrogen-provides-additional-information-roxadustat.

12 Akizawa T, Iwasaki M, Yamaguchi Y, Majikawa Y, Reusch M. Phase 3, randomized, double-blind, active-comparator (darbepoetin alfa) study of oral roxadustat in CKD patients with anemia on hemodialysis in Japan. J Am Soc Nephrol. 2020;31(7):1628-39. 